\title{
Petrology and Geodynamic Context of Metabasic Rocks of Nemba Complex in the West Congo Fold Belt (Republic of Congo)
}

\author{
Louis Marie Joachim Djama1, Ulrich Verne Matiaba Bazika1,2, Florent Boudzoumou1,2*, \\ Kinga Mouzeo ${ }^{1}$ \\ ${ }^{1}$ Université Marien Ngouabi, Département de Géologie, Brazzaville, Congo \\ ${ }^{2}$ Institut de Recherches en Sciences Exactes et Naturelles (IRSEN), Avenue de l'Auberge de Gascogne, Cité Scientifique, \\ (Ex-ORSTOM), Château d'Eau, Brazzaville, Congo \\ Email:1mdjama@hotmail.com, iletbazika@gmail.com, ${ }^{\star}$ florentboudzoumou@yahoo.fr, Kmouzeo@yahoo.fr
}

How to cite this paper: Djama, L.M.J., Bazika, U.V.M., Boudzoumou, F. and Mouzeo, K. (2018) Petrology and Geodynamic Context of Metabasic Rocks of Nemba Complex in the West Congo Fold Belt (Republic of Congo). International Journal of Geosciences, 9, 1-18. https://doi.org/10.4236/ijg.2018.91001

Received: October 20, 2017

Accepted: January 9, 2018

Published: January 12, 2018

Copyright $\odot 2017$ by authors and Scientific Research Publishing Inc. This work is licensed under the Creative Commons Attribution International License (CC BY 4.0).

http://creativecommons.org/licenses/by/4.0/

(C) (i) Open Access

\begin{abstract}
The West Congo belt contains Paleoproterozoic and Neoproterozoic Units which are deformed during Panafrican event. The Neoproterozoic Unit contains rift and post rift deposits. The rift formation is made of metabasite, volcanic and volcano-sedimentary rocks. The metabasite constitutes the Nemba Complex which is considered put into place in a continental or oceanic context. Samples from Nemba Complex collected along the "Congo ocean railway realignment" are analyzed. Major elements indicate a low potassic subalkaline affinity of the samples. Classification diagrams based of major elements shows that the rocks are a tholeiitic serie constituted of andesitic basalts, sub-alkaline andesitic basalts and a sub-alkaline to alkaline basalts. Some samples with a calc-alkaline signature indicate a crustal contamination. The traces elements plots indicate that the rocks are the MORB. The spectrum of multi-elements is characteristic of continental rocks with a lithospheric origin. This is an agreement with many works on the Nemba Complex and the alkali plutons and acid volcanic rocks associated which attribute there a rift context.
\end{abstract}

\section{Keywords}

West Congo, Neoproterozoic, Tholeiite, Andesitic Basalts, Basalts, MORB, Continental Rocks

\section{Introduction}

The west Congo belt (Figure 1) outcrops along the Atlantic eastern margin from South Gabon to the North Angola around $1400 \mathrm{~km}$ in length and 150 to $300 \mathrm{~km}$ 


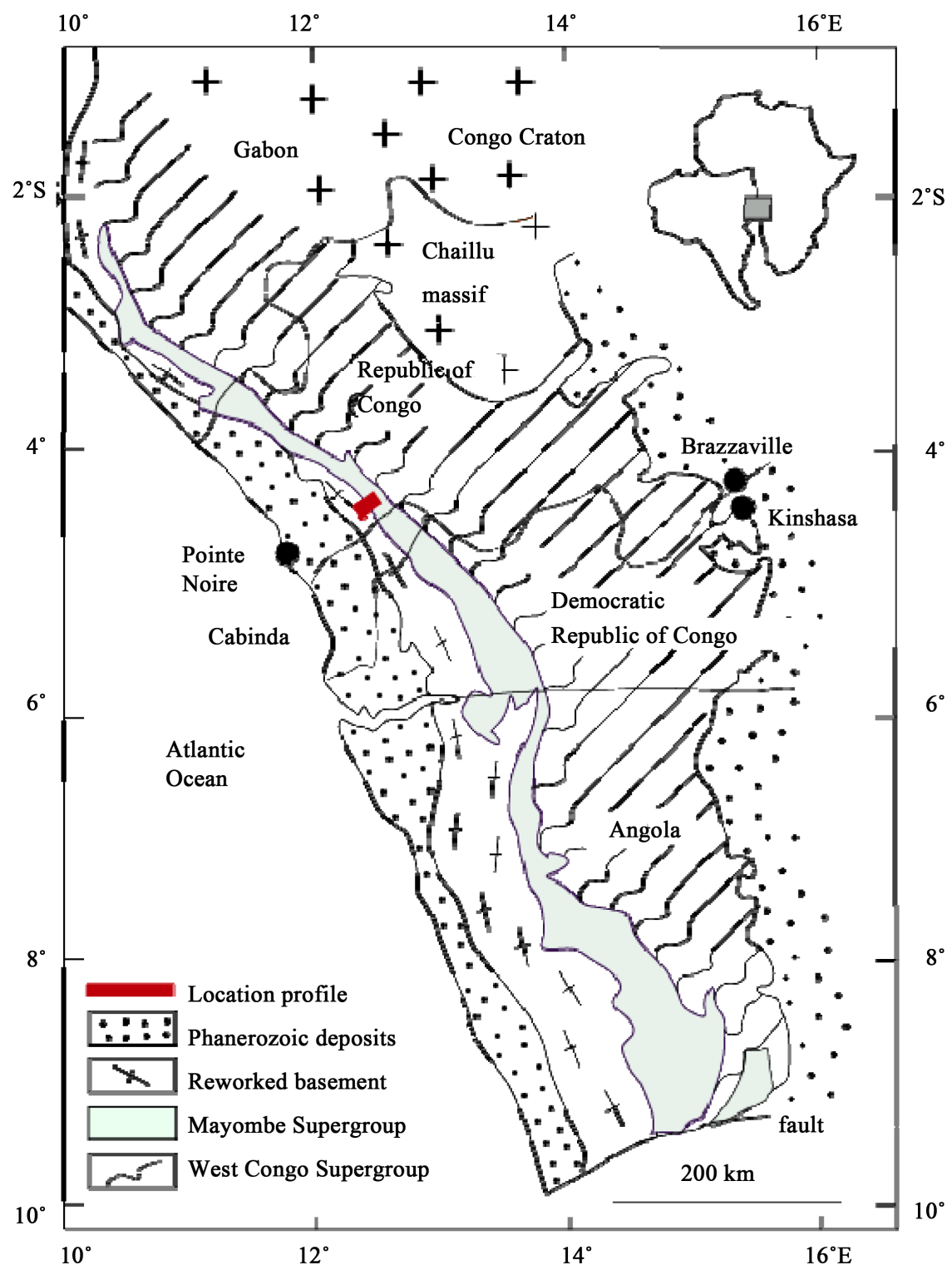

Figure 1. Main structural units of the West Congo modified after Boudzoumou and Trompette (1988), Affaton et al., 2015; Fullgraf et al., 2015. The inset in the upper right-hand corner illustrates the location of the Araçuai-West Congo orogen in Africa and South America.

in width. It represents the east part of the Araçuài-West Congo (A-WCO) belt. The west Congo belt is made of Paleoproterozoic and Neoproterozoic units deformed during Panafrican event. The Neoproterozoic formations are considered put into place, there is $1000 \mathrm{Ma}$, during the Rodinia breakup and deformed during Panafrican orogenesis [1] [2] [3].

The Araçùai belt includes arc magmatic type, syn to post collisional granites and a suture oceanic zone. Paleoproterozoic units of the west Congo belt are represented by Guéna formation and Loukoula and Bikossi groups [4] [5] called Premayombian Group [5]. It included granitoid and metasedimentary rocks 
reactive during Panafrican orogenic event. Neoproterozoic is made of rift and post rift deposits represented from bottom to top by Sounda Group (Nemba Complex, Kakamoeka Subgroup, Mvouti Subgroup) and Mayombe Group (Moussouva Subgroup, Lower Tillite formation and Louila Subgroup) [5].

The Nemba Complex and Kakamoeka Subgroup contain magmatic rocks which are used to determine the geodynamic context of the West Congo fold belt. The Nemba Complex is made of metabasite which is considered put into place in continental context [5] [6] [7] [8] [9] or in oceanic context [10]. For [10], the Nemba Complex is an ophiolite witness and the west Congo fold belt is a collisional belt which take place at around 1000 Ma. For [5] [6] [7] [8] [9], there is no evidence of oceanic suture and chemical analyses from Nemba Complex and its equivalent in RDC and Gabon, shows that rocks are continental tholeiite. These basic rocks and the acid rocks of Kakamoeka Subgroup and Neoproterozoic plutonic rocks associated are the witness of rifting stage.

In this study, we presented geochemistry data gotten on meta-basic rocks of Nemba Complex outcropping along the "Congo ocean railway realignment", between $\mathrm{Pk} 12$ and $\mathrm{Pk} 13$ from Nkougni to Dolisie town. Data gotten are showing that metabasite of Nemba Complex are a tholeiite which take place in continental context.

\section{Geological Setting of the Mayombe}

The geology of the Mayombe fold belt is made of added Paleoproterozoic and Neoproterozoic units.

The Paleoproterozoic is represented by Loémé Unit (or Guéna) in Congo, Kimezian Unit in DRC and Loukoula and Bikossi Groups. The Loémé Unit is made of tonalitic gneiss containing locally xenoliths of biotite gneiss and cross cutting by granitoids. The tonalitic gneiss are dated between $2129 \pm 68 \mathrm{Ma}$ and $1920 \pm 10 \mathrm{Ma}$ [4] [5] [11] [12] [13].

The Paleoproterozoic granitoids were dated between $2129 \pm 68 \mathrm{Ma}$ and $1952 \pm$ $77 \mathrm{Ma}$ [4] [14] [15] [16] [17]. The Loukoula Group is made of biotite paragneiss, orthogneiss, magnetite micaschists, schists, amphibolites and quartzites intruded locally by granitoids and dolerite. The zircon of paragneiss yield paleoproterozoic age [4] [5]. The Saras granodiorite which intrude the Loukoula Group is dated at $2000 \pm 80 \mathrm{Ma}$ by U/Pb on Zircon methods [13] and $1920 \pm 10 \mathrm{Ma}$ by $\mathrm{Pb} / \mathrm{Pb}$ single zircon methods [18]. The Bikossi Group is made of quartzite rich on magnetite and muscovite, locally conglomeratic at the lower part and micaschists, garnet schists and amphibolites in the upper part [8] [10] [19] [20]. Zircon of quartzite yielded age between $2200 \mathrm{Ma}$ and $2000 \mathrm{Ma}$ [4] [5]. The amphibolites whose some are described like metagabbro are considered by [5] as the syn-rift witness of Neoproterozoic age [SHRIMP age of $915 \pm 8$ Ma gotten from meta-gabbro zircon by [5] and are dissociated of Bikossi Group and attached in the lower part of Sounda Group of Fullgraf et al. (2015, [5]).

The Neoproterozoic consist of aulacogen type formation [8] [21] or rift and 
passive margin [5]. The rift formations are represented by Sounda Group containing from bottom to top, the Nemba Complex, the Kakamoeka and Mvouti Subgroups [5]. The Nemba Complex consists of metagabbros, metabasalts, amphibolites, and green schists. The Kakamoeka Subgroup is made of volcanic and volcano-sedimentary rocks (conglomerates, quartzites, graphitic schists, ignimbrites, rhyolites, tuffs, metabasic rocks) whose equivalent is dated in DRC between $920 \pm 8 \mathrm{Ma}$ and $917 \pm 14 \mathrm{Ma}$ [7]. TheMvouti Subgroup is constituted by black schists, chlorito-schists, quartzites, meta-arkoses and meta-sandstones.The post rift is represented by the Mayombe Group which contains the Moussouva Subgroup, the lower Tillite Formation and the Louila Subgroup. The Moussouva Subgroup is made of quartzites and schists. The lower Tillite is a glaciogenic formation with a black calcareous shale matrix and a doleritic sills intercalations in DRC. The Louila Subgroup contains meta-gres locally conglomeratic, quartzites, quartzo-schists and schists with intercalation of limestones and calcshists [8] [19] [22] [23]. The whole formation is intruded in the western domain by rifting contemporaneous granites (Mfoubou and Mont Kanda granites in Congo and Noqui granite in Democratic Republic of Congo).

The paleoproterozoic formations are deformed by eburnean phase generating a fold oriented NE-SW [8] [13] [20] which are reactive during the Panafrican orogenic events (West-Congolian) building a D1 and D2 folds oriented NW-SE associated respectively by cleavage ( $\mathrm{S} 1$ ) verging to the $\mathrm{NE}$ and crenulation cleavage (S2) often straighten and without clear verging [20] [21] [24]. A third tardy-orogenic phase characterized by a second crenulation cleavage (S3) oriented NE-SW folding a D1 and D2 structures is described in the Loémé Formation (or Guéna Subgroup) [8] [21]. In the Mayombe, deformation is also accompanied by thrust pulling the formation of internal domain stacked upon the formation of the external domain. The intensity of deformation decreases from SW (internal domain) to the NE of the belt (external domain).

The metamorphism is an amphibolite facies grade in the internal domain. It decreases to the NE where it becomes greenschist facies grade in the external domain and anchizone facies grade near the adjoining Niari basin.

\section{Sampling}

21 samples are collected along the "Congo ocean railway realignment", between the Pk 12 and Pk 13 from Nkougni to Dolisie town. In this area outcrops largely the Nemba Complex formation with intercalation in small part of Loukoula Subgroup.

The Nemba Complex formation is essentially made of amphibolites and greenschists (Figures 2(a)-(c)). The amphibolites are in massive hard beds and the greenschists are strongly cleavage. Locally the two facies contains veins filled by quartz or calcite. The associated fold are long flank and short flank and a slight $\mathrm{NE}$ verging. The whole rocks shown a crenulation cleavage (S2) strongly straightens. The quartzo-schists (Figure $2(\mathrm{~d})$ ) are essentially made of quartzo-feldspathic levels alternating with wealthy muscovite levels. The shows also a 


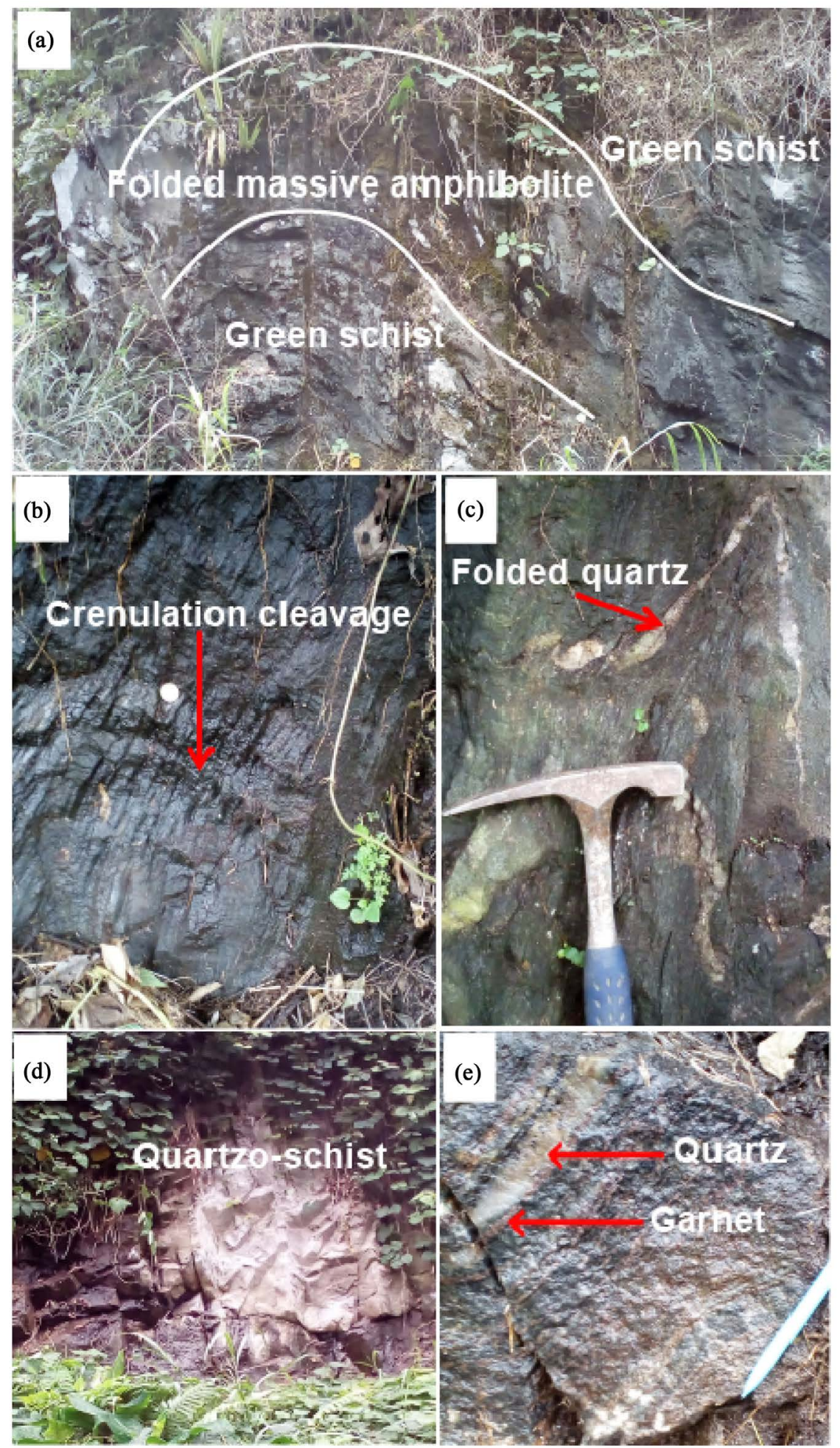

Figure 2. Outcrops of basic Nemba Complex formation and Loukoula quartzo-schist along the "Congo ocean railway realignment": (a)-(c) metabasite rocks; (d) quartzo-schist; (e) migmatite bearing garnet.

crenulation cleavage (S2) oriented $\mathrm{N} 162^{\circ}-88^{\circ}$ toward the SW and $\mathrm{N} 160^{\circ}-77^{\circ}$ toward the NE. 
Locally between the Nemba Complex formation and the quartzo-schists is measured an inverse fault with direction of $\mathrm{N} 156^{\circ}-25^{\circ}$ toward to the SW. A migmatite bearing garnet on centimetric layer (Figure $2(e)$ ) has been also observed on place.

\section{Analytical Procedures}

Geochemical analyses of major, trace and rare earth elements were done to evaluate the petrogenesis of rocks. Major, trace and REE elements were determined after powdered samples in fusion with $\mathrm{LiBO}_{2}$ and dilute by $\mathrm{HNO} 3$ and performed by inductively coupled plasma-mass spectrometry (ICP-MS) and inductively coupled plasma atomic emission spectrometry (ICP-AES) in the "Centre de Recherches Pétrographique et Géochimique” (CRPG) of Nancy, France.

\section{Results}

\subsection{Microscopic Description}

Samples of Nemba Complex show lepidoblastic, lepido-granoblastic and grano-lepidoblastic texture.

The amphibolites (Figures $3(\mathrm{a})-(\mathrm{c})$ ) contain green hornblende, biotite,
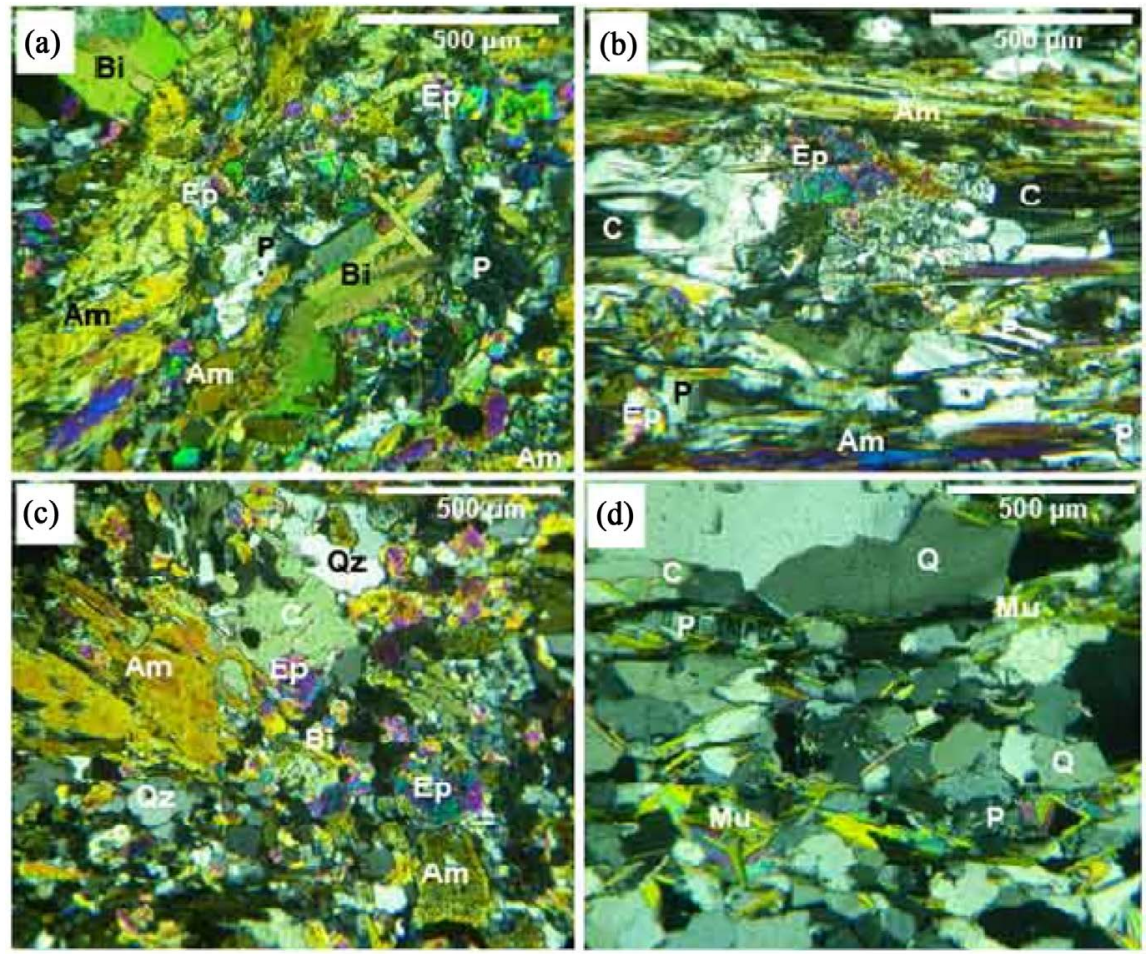

Figure 3. Photomicrographs of basic rocks of Nemba Complex formation and quartzo-schist of Loukoula Group: (a) sample NE11, basalt showing biotite, amphibole, plagioclase and epidote; (b) sample N20, andesitic basalt showing chlorite substitute amphibole in some case; (c) samples NE21, andesitic basalt containing accessory calcite; (d) Sample N22, quartzo-schist with quartz, feldspar, muscovite and accessory calcite. Am-Amphibolite, Bi-Biotite, C-Calcite, Ep-Epidote, $\mathrm{Mu}-$ Muscovite, $\mathrm{P}$-Plagioclase, Q-Quartz. 
chlorite, epidote, K-feldspar, plagioclase and accessory quartz, calcite, sulphide and opaque mineral in some samples. Chlorite often replaces the biotite. In the sample with crenulation cleavage, chlorite flats occupy S2 cleavage which cut the amphibole. In some samples the core of k-feldspar displays a damourite alteration. Quartz, plagioclases and calcite crystallize also in the fissure and the shadow pressure developed in the rock. The will characterize a probable circulation zone of hydrothermal fluids. The greenschists contain chlorite, biotite, epidote, quartz, plagioclase and accessory amphibole, calcite, apatite, zircon, and pyrite. Like the amphibolite, quartz, feldspars and accessory the calcite occurs in the fissures and the shadow pressure. The quartzo-schists (or paragneiss) bear a granoblastic texture (Figure 3(d)). They contain quartz, muscovite, biotite, plagioclases and accessory epidote and calcite.

\subsection{Geochemistry and Petrographic Classification}

Representative analyses are given in Table 1.

Table 1. Major and trace elements analysis.

\begin{tabular}{|c|c|c|c|c|c|c|c|c|c|c|c|c|c|c|c|c|c|c|c|c|c|}
\hline $\mathrm{SiO}_{2}$ & 51.17 & 45 & 46.84 & 46.31 & 47.83 & 50.54 & 50.28 & 52.41 & 49.85 & 49.34 & 49.56 & 57.71 & 49.95 & 42.11 & 48.11 & 51.12 & 40.25 & 51.31 & 45.71 & 52.68 & 41.86 \\
\hline $\mathrm{AL}_{2} \mathrm{O}_{3}$ & 14.49 & 16.07 & 14.9 & 15.94 & 15.26 & 15.25 & 14.23 & 12.52 & 15.32 & 15.16 & 15.26 & 11.13 & 15.87 & 15.2 & 14.24 & 15.63 & 18.19 & 15.3 & 14.07 & 14.73 & 14.99 \\
\hline $\mathrm{Fe} 2 \mathrm{O} 3$ & 9.43 & 12.48 & 11.82 & 11.81 & 11.19 & 9.97 & 10.92 & 10.34 & 10.32 & 11.01 & 10.57 & 9.08 & 9.76 & 16.56 & 13.73 & 9.98 & 13.85 & 9.91 & 17.14 & 11.5 & 18.19 \\
\hline $\mathrm{MnO}$ & 0.14 & 0.18 & 0.17 & 0.17 & 0.16 & 0.16 & 0.17 & 0.17 & 0.16 & 0.18 & 0.17 & 0.13 & 0.14 & 0.22 & 0.21 & 0.16 & 0.19 & 0.15 & 0.24 & 0.19 & 0.26 \\
\hline $\mathrm{MgO}$ & 3.55 & 8.61 & 6.81 & 8.26 & 6.95 & 5.61 & 8.93 & 6 & 7.74 & 8.33 & 7.48 & 3.59 & 6.6 & 3.26 & 6.44 & 5.72 & 2.66 & 5.22 & 5.99 & 6.11 & 6.1 \\
\hline $\mathrm{CaO}$ & 15 & 9.72 & 12.06 & 8.99 & 12.16 & 12.43 & 7.4 & 13.85 & 8.17 & 7.62 & 8.78 & 13.37 & 9.56 & 16.28 & 11.15 & 8.91 & 20.02 & 11.12 & 9.69 & 7.3 & 11.36 \\
\hline $\mathrm{Na}_{2} \mathrm{O}$ & 1.09 & 2.29 & 2.17 & 3.02 & 2.04 & 2.16 & 3.17 & 0.76 & 4.1 & 3.82 & 4.06 & 0.39 & 4 & 0.64 & 1.9 & 4.44 & 0.31 & 3.79 & 2.87 & 3.4 & 2.38 \\
\hline $\mathrm{K}_{2} \mathrm{O}$ & 0.09 & 0.61 & 0.59 & 0.64 & 0.25 & 0.33 & 0.19 & 0.12 & 0.41 & 0.47 & 0.23 & 0.1 & 0.14 & 0.19 & 0.33 & 0.58 & 0.08 & 0.16 & 0.4 & 1.64 & 0.41 \\
\hline $\mathrm{TiO}_{2}$ & 1.29 & 1.31 & 1.42 & 1.33 & 0.84 & 0.81 & 0.8 & 0.97 & 0.97 & 0.84 & 0.84 & 1.12 & 0.81 & 2.1 & 1.24 & 1.07 & 0.88 & 1.05 & 2.26 & 0.93 & 2.49 \\
\hline $\mathrm{P}_{2} \mathrm{O}_{5}$ & 0.26 & 0.23 & 0.24 & 0.23 & 0.22 & $<5$ & $<5$ & $<5$ & $<5$ & $<5$ & $<5$ & $<5$ & $<5$ & 0.39 & $<5$ & $<5$ & $<5$ & $<5$ & 0.33 & $<5$ & 0.27 \\
\hline PF & 2.02 & 2.83 & 1.74 & 2.62 & 1.88 & 1.57 & 2.45 & 1.35 & 2.12 & 2.2 & 1.7 & 2.05 & 2.35 & 2.05 & 1.29 & 1.02 & 2.28 & 1.03 & 0.57 & 1.01 & 0.73 \\
\hline Total & 98.52 & 99.32 & 98.76 & 99.3 & 98.79 & 98.83 & 98.52 & 98.52 & 99.15 & 98.98 & 98.65 & 98.66 & 99.19 & 98.98 & 98.63 & 98.62 & 98.17 & 99.04 & 99.26 & 99.48 & 99.02 \\
\hline Sc & 37.32 & 38.91 & 40.01 & 38.25 & 32.81 & 34.83 & 36.52 & 42.61 & 40.19 & 38 & 39.31 & 34.63 & 38.34 & 42.48 & 40.74 & 31.78 & 26.45 & 31.34 & 43.52 & 37 & 47.48 \\
\hline As & 0.39 & 0.26 & 0.37 & 0.38 & 0.27 & 0.24 & $<20$ & $<20$ & 0.37 & $<20$ & 0.25 & 0.35 & 0.49 & 0.78 & 0.27 & 0.42 & 0.93 & 0.27 & 0.29 & $<20$ & 0.28 \\
\hline $\mathrm{Ba}$ & 31.6 & 393 & 359 & 417 & 137 & 201 & 82.8 & 21.7 & 254 & 314 & 94.7 & 24.8 & 49.4 & 14.3 & 130 & 336 & 23.3 & 37.3 & 43.7 & 741 & 55.9 \\
\hline $\mathrm{Be}$ & 0.32 & 0.41 & 0.52 & 0.47 & 0.52 & 0.41 & 0.37 & 0.41 & 0.41 & 0.45 & 0.56 & 0.37 & 0.39 & 0.82 & 0.55 & 0.37 & 0.37 & 0.58 & 1.14 & 0.76 & 1.15 \\
\hline $\mathrm{Bi}$ & $<5$ & $<5$ & $<5$ & $<5$ & $<5$ & $<5$ & $<5$ & $<5$ & $<5$ & $<5$ & $<5$ & $<5$ & $<5$ & 0.06 & $<5$ & $<5$ & $<5$ & $<5$ & $<5$ & $<5$ & 0.05 \\
\hline $\mathrm{Cd}$ & 0.12 & 0.06 & 0.11 & 0.08 & 0.11 & 0.11 & 0.05 & 0.1 & 0.05 & 0.07 & 0.07 & 0.08 & 0.11 & 0.22 & 0.1 & 0.08 & 0.14 & 0.1 & 0.11 & 0.09 & 0.16 \\
\hline $\mathrm{Ce}$ & 23.9 & 24.9 & 26 & 24.2 & 43.1 & 28.8 & 27.7 & 31.7 & 34.2 & 28.6 & 28.2 & 38.8 & 21.5 & 61.2 & 29.5 & 25.3 & 25.1 & 26.5 & 55.9 & 43.1 & 70.4 \\
\hline Co & 33.9 & 53.9 & 45.4 & 51.1 & 49.4 & 36.5 & 52.3 & 38.1 & 56.4 & 49.2 & 44.8 & 24.4 & 53.5 & 39.4 & 51.4 & 44.8 & 19 & 39.3 & 55.4 & 47 & 58.3 \\
\hline
\end{tabular}




\section{Continued}

\begin{tabular}{|c|c|c|c|c|c|c|c|c|c|c|c|c|c|c|c|c|c|c|c|c|c|}
\hline $\mathrm{Cr}$ & 196 & 208 & 197 & 204 & 274 & 306 & 325 & 352 & 297 & 342 & 341 & 121 & 94.3 & 87.2 & 77.3 & 118 & 114 & 139 & 78.4 & 53.3 & 84.3 \\
\hline Cs & 0.07 & 03 & 1.06 & 1.19 & 0.28 & 0.41 & 0.17 & 0.05 & 0.61 & 0.61 & 0.19 & 0.04 & 0.02 & $<20$ & 0.08 & 0.28 & 0.02 & 0.02 & $<20$ & 45 & 0.06 \\
\hline $\mathrm{Cu}$ & 22.2 & 1.3 & 301 & 51.5 & 8.4 & 93.2 & 58.2 & $<8$ & $<8$ & 29.7 & 34.1 & 36.4 & 6.8 & 1408 & 184 & 18.1 & 217 & 36.2 & 127 & 110 & 48 \\
\hline Dy & 3 & 3.27 & 3.44 & 3.26 & 3.61 & 3.06 & 3.14 & 3.8 & 3.73 & 3.35 & 3.26 & 4.23 & 3.07 & 7.26 & 3.92 & 3.35 & 2.88 & 3.19 & 6.56 & 4.18 & 7.58 \\
\hline Er & 1.83 & 1.94 & 2.05 & 1.94 & 2.01 & 1.83 & 1.86 & 2.18 & 2.24 & 2.01 & 1.97 & 2.57 & 1.87 & 4.26 & 2.29 & 1.94 & 1.67 & 1.77 & 3.8 & .46 & 4.41 \\
\hline $\mathrm{Eu}$ & 1.1 & 1.13 & 1.1 & 1.03 & 1.26 & 1.04 & 0.872 & 1.11 & 1.17 & 1.06 & 1.04 & 1.3 & 0.947 & 2.22 & 1.26 & 1.56 & 1.58 & 1.38 & 1.67 & 1.23 & 16 \\
\hline $\mathrm{Ga}$ & 18.7 & 15.6 & 16.3 & 15.1 & 15.8 & 18.6 & 13.1 & 18.5 & 14.7 & 16 & 16.5 & 17.5 & 16.1 & 31.2 & 21.1 & 17.4 & 35.4 & 15.8 & 21.8 & 19.8 & 25.5 \\
\hline $\mathrm{Gd}$ & 2.82 & 3.08 & 3.11 & 2.94 & 3.62 & 2.88 & 2.88 & 3.31 & 3.41 & 2.99 & 2.92 & 3.9 & 2.72 & 6.74 & 3.65 & 3.25 & 2.98 & 3.14 & 6.06 & .98 & 7.27 \\
\hline $\mathrm{Ge}$ & 1.49 & 1.21 & 1.4 & 1.12 & 1.35 & 1.57 & 1.01 & 1.91 & 1.13 & 1.09 & 1.21 & 1.67 & 1.34 & 2.47 & 1.6 & 1.33 & 2.6 & 1.4 & 1.41 & 1.35 & 1.64 \\
\hline Hf & 1.61 & 1.62 & 1.76 & 1.67 & 1.69 & 1.72 & 2.03 & 2.49 & 2.52 & 2.14 & 2.12 & 2.92 & 1.86 & 5.1 & 2.54 & 1.85 & 1.55 & 1.78 & 5.21 & 3.18 & 5.77 \\
\hline Ho & 0.67 & 0.715 & 0.763 & 0.713 & 0.756 & 0.673 & 0.68 & 0.822 & 0.803 & 0.728 & 0.718 & 0.943 & 0.681 & 1.57 & 0.842 & 0.717 & 0.626 & 0.671 & 1.4 & 0.895 & 1.62 \\
\hline In & 0.06 & 0.06 & 0.07 & 0.06 & 0.05 & 0.05 & 0.05 & 0.06 & 0.06 & 0.06 & 0.06 & 0.07 & 0.06 & 0.13 & 0.08 & 0.07 & 0.07 & 0.06 & 0.09 & 0.06 & 0.11 \\
\hline $\mathrm{La}$ & 11.5 & 1.5 & 12 & .1 & 21 & 14 & 13.4 & 15.2 & 6.7 & 13.7 & 13.5 & 18.6 & 9.75 & 29.6 & 13.9 & 11.2 & 11.4 & 11.4 & 25.1 & 0.8 & 33.4 \\
\hline $\mathrm{Lu}$ & 0.278 & 0.285 & 0.305 & 0.286 & 0.289 & 0.272 & 0.277 & 0.323 & 0.333 & 0.295 & 0.293 & 0.378 & 0.282 & 0.642 & 0.34 & 0.285 & 0.241 & 0.251 & 0.561 & 0.354 & 0.63 \\
\hline Mo & $<5$ & $<5$ & $<5$ & 0.58 & $<5$ & 0.51 & $<5$ & 0.9 & $<5$ & $<5$ & $<5$ & $<5$ & $<5$ & $<5$ & $<5$ & $<5$ & 0.52 & $<5$ & $<5$ & $<5$ & $<5$ \\
\hline $\mathrm{Nb}$ & 11.2 & 11.2 & 12.4 & 11.3 & 10.5 & 5.22 & 4.27 & 5.39 & 5.43 & 4.4 & 4.45 & 6.29 & 3.38 & 12.5 & 5.43 & 4.44 & 3.55 & 4.13 & 12.8 & 21 & 13 \\
\hline $\mathrm{Nd}$ & 12.5 & 13.4 & 13.7 & 12.5 & 19.2 & 13.8 & 13 & 15.1 & 16.1 & 13.5 & 13.3 & 18 & 11.4 & 30.4 & 15.4 & 14.3 & 14 & 15 & 27.2 & 8.8 & 3.9 \\
\hline $\mathrm{Ni}$ & 188 & 427 & 133 & 192 & 190 & 184 & 150 & 145 & 158 & 133 & 175 & 100 & 332 & 324 & 186 & 451 & 538 & 239 & 98.6 & 63.2 & 97.1 \\
\hline $\mathrm{Pb}$ & 3.35 & 1.65 & 2.57 & 1.55 & 3.15 & 4.67 & 2.76 & 5.04 & 2.2 & 1.8 & 2.72 & 4.53 & 4.03 & 12.1 & 3.78 & 3.97 & 14.7 & 5.01 & 3.1 & 4.8 & 5.45 \\
\hline $\operatorname{Pr}$ & 2.92 & 3.09 & 3.19 & 2.98 & 4.94 & 3.4 & 3.23 & 3.71 & 3.97 & 3.37 & 3.31 & 4.48 & 2.64 & 7.28 & 3.59 & 3.24 & 3.17 & 3.41 & 6.64 & 4.81 & 8.24 \\
\hline $\mathrm{Rb}$ & 0.79 & 12.6 & 12.9 & 13.7 & 3.78 & 5.83 & 2.45 & 0.74 & 8.61 & 10 & .1 & 0.87 & 1.06 & 0.82 & 4.81 & 16 & 0.89 & 1.43 & 3.03 & 51.5 & 3.74 \\
\hline $\mathrm{Sb}$ & 0.07 & 0.07 & 0.08 & 0.05 & 0.05 & 0.06 & $<5$ & 0.08 & 0.07 & $<20$ & $<20$ & 0.07 & 0.07 & 0.07 & $<20$ & 0.06 & 0.05 & $<5$ & $<20$ & $<0.05$ & $<20$ \\
\hline $\mathrm{Sm}$ & 2.78 & 3.03 & 3.14 & 2.9 & 3.85 & 2.96 & 2.89 & 3.31 & 3.51 & 3.01 & 2.97 & 4.02 & 2.7 & 7 & 3.64 & 3.39 & 3.18 & 3.46 & 6.12 & 4.08 & 7.56 \\
\hline Sn & $<15$ & $<15$ & $<15$ & $<15$ & $<15$ & $<15$ & $<15$ & $<15$ & $<15$ & $<15$ & $<15$ & $<15$ & $<15$ & 2.16 & $<15$ & $<15$ & $<15$ & $<15$ & 2.01 & 15 & $<15$ \\
\hline $\mathrm{Sr}$ & 363 & 206 & 23 & 161 & 579 & 662 & 22 & 44 & 23 & 169 & 216 & 523 & 40 & 7 & 30 & 226 & 1150 & 384 & 163 & 50 & 04 \\
\hline $\mathrm{Ta}$ & 0.81 & 0.81 & 0.9 & 84 & 0.65 & 0.34 & 0.28 & 0.34 & 0.35 & 0.29 & 0.29 & 0.4 & 0.21 & 0.82 & 0.34 & 0.31 & 0.23 & 0.28 & 0.75 & 0.44 & 0.84 \\
\hline $\mathrm{Tb}$ & 0.462 & 0.498 & 0.52 & 0.487 & 0.563 & 0.47 & 0.474 & 0.572 & 0.565 & 0.504 & 0.491 & 0.657 & 0.466 & 1.12 & 0.605 & 0.529 & 0.471 & 0.507 & 1.01 & 0.654 & 1.19 \\
\hline Th & 0 & 0.87 & 0 & 0.89 & 1.2 & 0. & 1.78 & 2.17 & 2.17 & 1.87 & 1.81 & 2.6 & 0.61 & 2.67 & 1.34 & 0.65 & .45 & 0.5 & 3.06 & .47 & 31 \\
\hline $\mathrm{Tm}$ & 265 & 0.274 & 0.296 & 0.278 & 0.282 & 0.26 & 0.269 & 0.314 & 0.321 & 0.287 & 0.28 & 0.366 & 0.266 & 0.602 & 0.327 & 0.273 & 0.233 & 0.245 & 0.534 & 0.347 & .62 \\
\hline $\mathrm{U}$ & 0.26 & 0.19 & 0.21 & 0.16 & 0.26 & 0.15 & 0.23 & 0.23 & 0.24 & 0.21 & 0.2 & 0.4 & 0.11 & 0.51 & 0.19 & 0.15 & 0.11 & 0.11 & 0.48 & 1.15 & 0.5 \\
\hline $\mathrm{V}$ & 237 & 263 & 26 & 261 & 204 & 235 & 207 & 233 & 22 & 227 & 24 & 38 & 7 & 360 & 295 & 207 & 265 & 197 & 362 & 36 & 95 \\
\hline W & $<10$ & $<10$ & 10 & 10 & $<10$ & $<10$ & $<10$ & $<10$ & $<10$ & $<10$ & $<10$ & 10 & 10 & $<10$ & $<10$ & $<10$ & $<10$ & $<10$ & $<10$ & $<10$ & 10 \\
\hline $\mathrm{Y}$ & 17.1 & 18.5 & 19.4 & 18.2 & 19.8 & 17.4 & 18.1 & 21 & 20.9 & 18.8 & 18.5 & 24.2 & 17.4 & 39.9 & 22.1 & 18.7 & 16.4 & 17.4 & 35.7 & 23 & 42. \\
\hline $\mathrm{Yb}$ & 1.82 & 1.86 & 2 & 1.89 & 1.88 & 1.78 & 1.84 & 2.1 & 2.17 & 1.94 & 1.91 & 2.46 & 1.82 & 4.15 & 2.21 & 1.85 & 1.55 & 1.62 & 3.63 & 2.36 & 4.16 \\
\hline $\mathrm{Zn}$ & 34.9 & 93 & 81 & 92.1 & 76.1 & 58.9 & 104 & 60.2 & 87 & 102 & 85.9 & 36.7 & 72.1 & 87.1 & 106 & 71.8 & 30.4 & 58.6 & 154 & 107 & 160 \\
\hline $\mathrm{Zr}$ & 63.1 & 64.6 & 74.1 & 64.9 & 70 & 69.6 & 78.8 & 96.4 & 97 & 81.3 & 79.9 & 114 & 74.9 & 205 & 102 & 70.6 & 59.6 & 67 & 217 & 115 & 2 \\
\hline
\end{tabular}




\subsubsection{Major Elements}

Major elements (Table 1) give value $\mathrm{SiO}_{2}$ (40.25\% - 57.71\%), $\mathrm{Al}_{2} \mathrm{O}_{3}(11.13 \%$ $18.19 \%), \mathrm{MgO}\left(2.66 \%\right.$ - 9.37\%), $\mathrm{CaO}(7.3 \%-20.02 \%), \mathrm{Na}_{2} \mathrm{O}(0.39 \%-4.44 \%)$ and $\mathrm{TiO}_{2}(0.61 \%-2.42 \%)$. The LOI values $(0.57-2.83)$ are low to medium and rendered a partially to medium weathering which could lead a secondary hydration of minerals. $\mathrm{SiO}_{2}, \mathrm{MgO}$ contents and total alkalinity $(0.31-4.44)$ displays a low-K sub alkaline affinity. These rocks rich in $\mathrm{Al}_{2} \mathrm{O}_{3}$ and $\mathrm{CaO}$ and lightly poor in $\mathrm{TiO}_{2}$ show a character of orogenic lavas [25]. The classification diagram based on major elements [26] [27] [28] plots the rocks in tholeiitic serie (Figures $4(\mathrm{a})-(\mathrm{c}))$.

The tholeiitic series is constituted of andesitic basalts (NE 16, 17, 21, 26, 27, 31, $3518,19,20,25,30,32,34)$ certain of high-Ti (NE 26, 27, 34, 35), a sub-alkaline andesitic basalts (NE 15,33) and sub-alkaline to alkaline basalts (NE 10,11, 12, 13, 14), certain of high $\mathrm{Mg}$ (NE 11,13,14). The Harker diagram (Figure 5) of major elements shows a dispersion data with weak correlation coefficient. The $\mathrm{K}_{2} \mathrm{O}$ vs. $\mathrm{MgO}$ displays a linear correlation in the majority of the samples [except

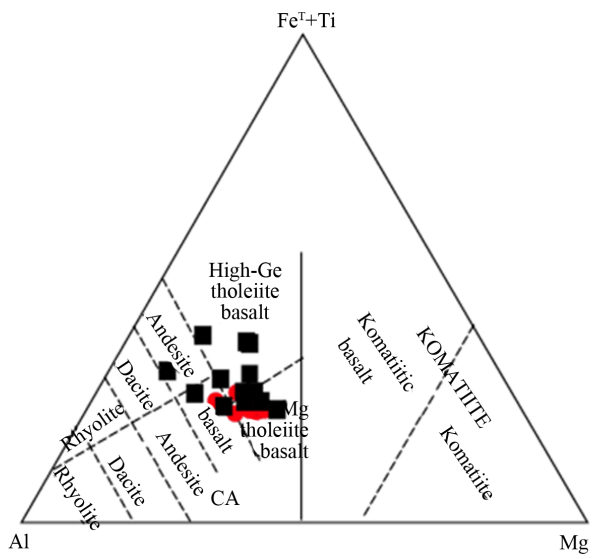

(a)

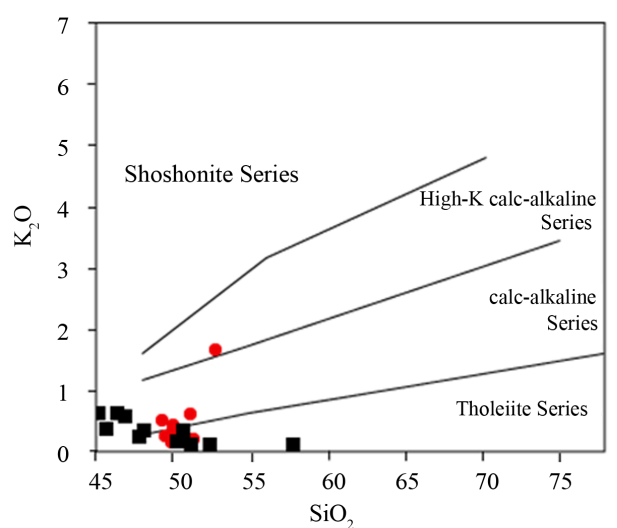

(b)

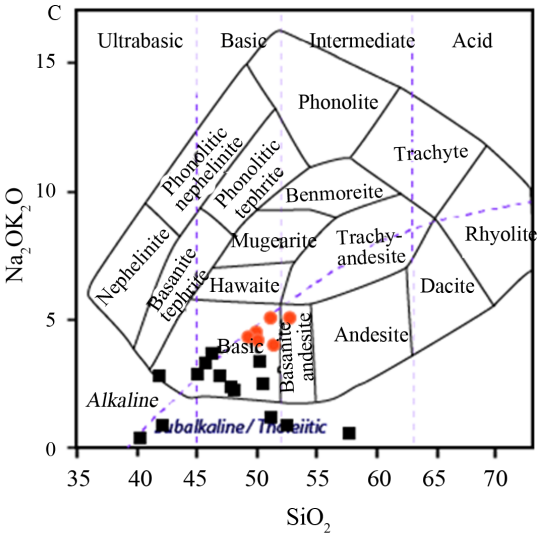

(c)

Figure 4. Geochemical characterization of Nemba samples: (a) $\left(\mathrm{FeOt}+\mathrm{TiO}_{2}\right)-\mathrm{Al}_{2} \mathrm{O}_{3}-$ $\mathrm{MgO}$ diagram de Jensen (1976); (b) $\mathrm{K}_{2} \mathrm{O}$ vs. $\mathrm{SiO}_{2}$ diagram de Peccerillo and Taylor (1976); (c) $\mathrm{Na}_{2} \mathrm{O}+\mathrm{K}_{2} \mathrm{O}$ vs. $\mathrm{SiO}_{2}$ diagram de Tas (Cox et al., 1979). 

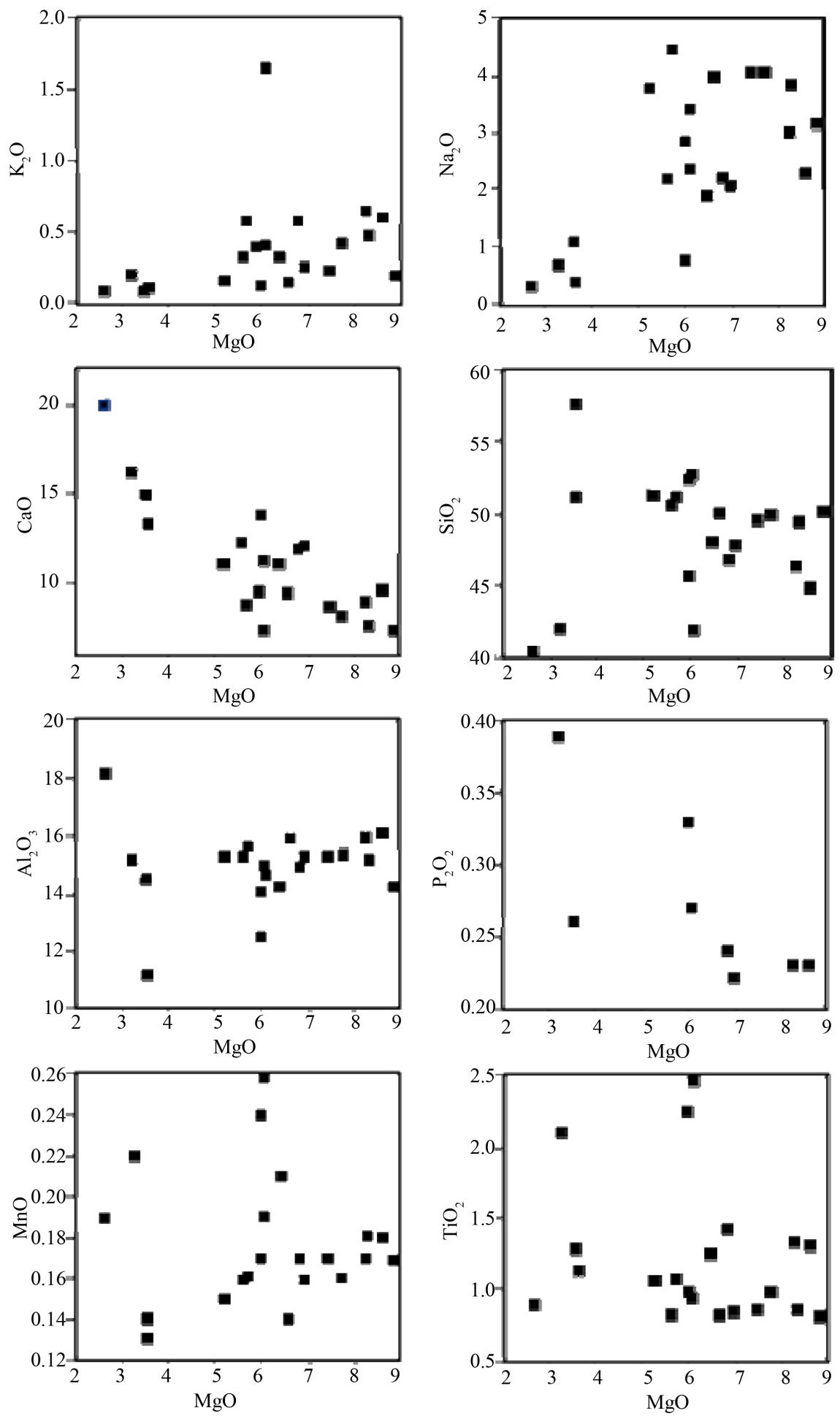

Figure 5. Harker diagrams of some major elements of Nemba Complex showing dispersal elements.

the $\mathrm{NE} 34$ which is richer in $\left.\mathrm{K}_{2} \mathrm{O}(1.49 \%)\right]$. The $\mathrm{Na}_{2} \mathrm{O}$ vs. $\mathrm{MgO}$ shows a positive correlation in the rocks with a tholeiitic signature although rocks with calc-alkaline signature don't indicate any correlation. The $\mathrm{CaO}$ vs. $\mathrm{MgO}$ display a negative 
correlation. The others major elements like the $\mathrm{SiO}_{2}, \mathrm{Al}_{2} \mathrm{O}_{3}, \mathrm{MnO}, \mathrm{TiO}_{2}$ and $\mathrm{P}_{2} \mathrm{O}_{5}$ vs. $\mathrm{MgO}$ displays a dispersal data with a weak coefficient of correlation.

\subsubsection{Traces Elements}

Trace elements diagram $\mathrm{Zr} / \mathrm{TiO}_{2}$ vs. $\mathrm{Nb} / \mathrm{Y}$ from [29] displays a predominance of the rocks in the basalt/andesite domain and the minority of rocks in the sub-alkali basalts domain (Figure 6). Ti vs. Zr diagram of [30] plots the samples in MORB (NE 10,11, 12, 13, 27, 30, 31, 32), Island Arc lavas (NE 14, 15, 16, 17, 18, 19, 20, $21,25,34)$, and intraplate (NE 26, 33, 35) domains (Figure 7(a)). The Th/Yb vs. $\mathrm{Nb} / \mathrm{Yb}$ diagram of [31] plots some samples in the EMORB domain while the major part is situated above this domain with a weak dispersion (Figure 7(b)). Any sample fall in the N-MORB and OIB-MORB. Compatible trace elements like $\mathrm{Co}, \mathrm{Cr}$ shows a positive correlation with the $\mathrm{MgO}$, while the Ni didn't show any correlation. The mobile incompatible elements, $\mathrm{Rb}, \mathrm{Sr}$ and $\mathrm{Ce}$ don't show any significant tendency. On the other hand, Ba vs. MgO show a $\Sigma$ positive correlation. Immobile incompatible elements, $\mathrm{Nb}, \mathrm{Zr}, \mathrm{Nd}, \mathrm{Th}, \mathrm{Y}$ and $\mathrm{Hf}$ presents one majority group with a weakly concentration and a group of tree samples with a high concentration. REE normalized to chondrites [32] displays sample enriched relative to LREE/HREE $\left((\mathrm{La} / \mathrm{Yb})_{\mathrm{N}}: 3.57-7.49\right)$ with a majority group weakly poor in HREE and accompanied of Eu positive anomaly (except NE 34 which contains Eu negative anomaly) and a group of tree samples(NE 26, 33, 35) showing higher REE $(\Sigma \mathrm{REE}=149$ - 183) concentration and contains Eu negative anomaly (Figure 8(a)). Spectrum of multi-elements normalized at primitive mantle of [33] shows also the bimodal comportment of samples with

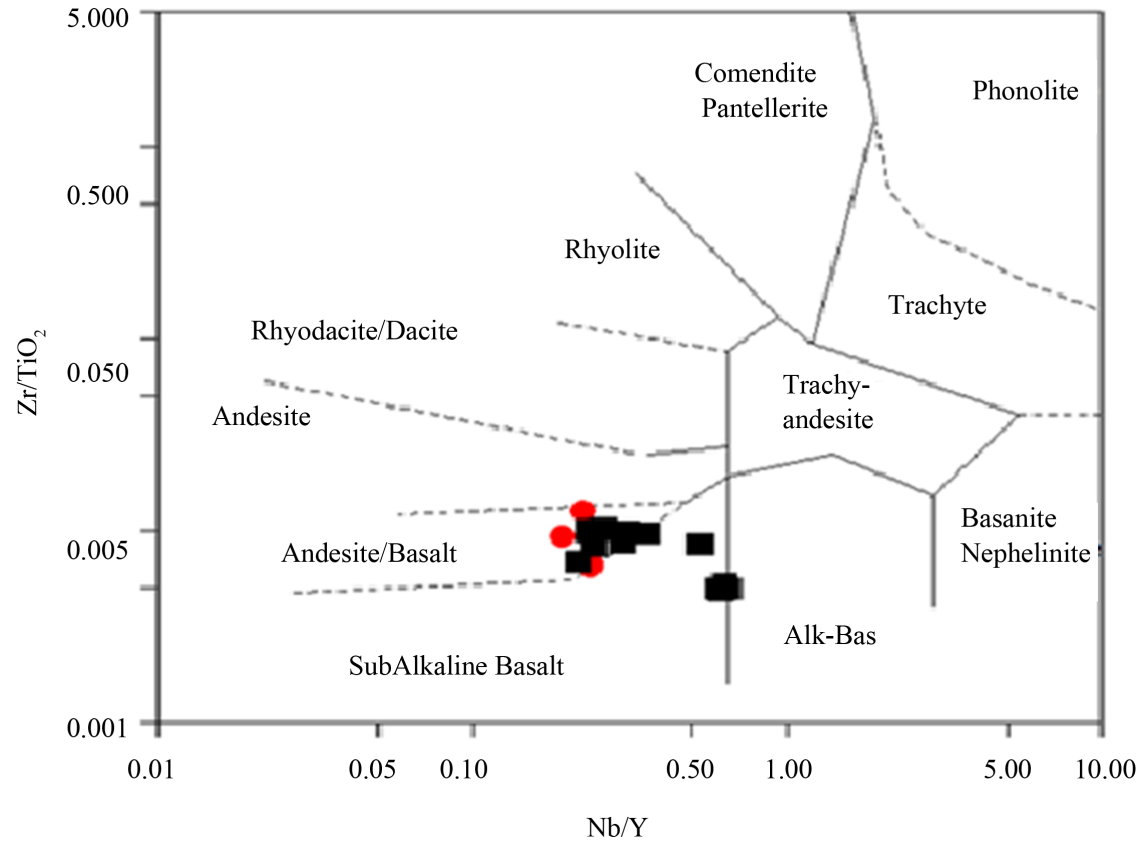

Figure 6. $\mathrm{Zr} / \mathrm{TiO}_{2}$ vs. $\mathrm{Nb} / \mathrm{Y}$ diagram of Winchester and Floyd (1977) showing a majority samples in the andesite/basalt and a few samples in subalkaline basalt. 


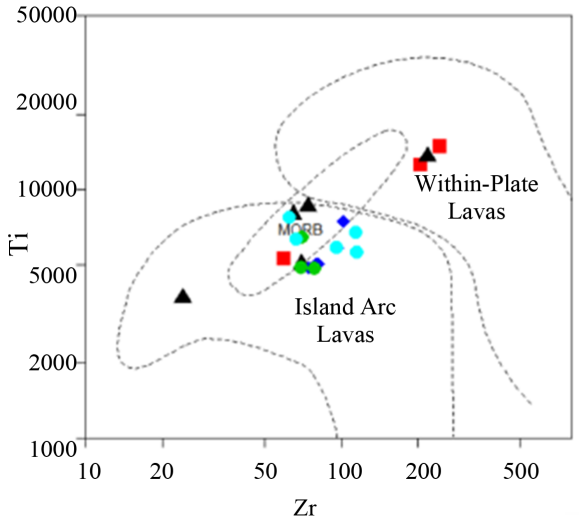

(a)

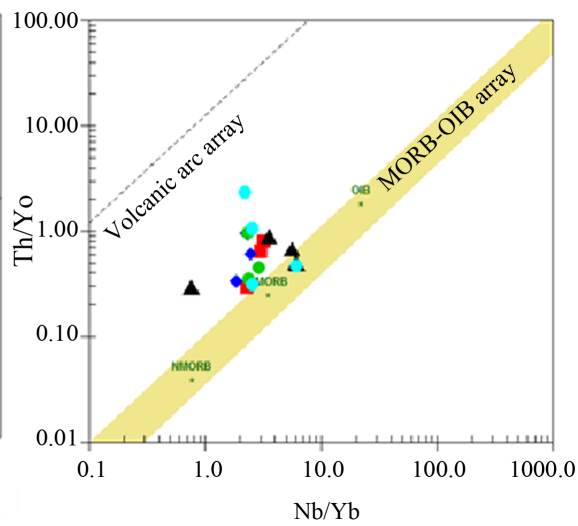

(b)

Figure 7. Plotted samples in discrimination diagrams; (a) Ti vs. Zr of Pearce (1982) showing a distribution of samples in Island Arc Lavas, MORB and Within Plate Lavas; (b) $\mathrm{Th} / \mathrm{Yb}$ vs. Nb/Yb of Pearce (2008) displaying a distribution in the E-MORB domain and in the top in arc array Volcanic domain.

the majority group which possess a $\mathrm{Ba}, \mathrm{Pb}, \mathrm{Sr}$ positive anomalies and $\mathrm{Rb}, \mathrm{Zr}, \mathrm{Ti}$, $\mathrm{Nb}$-Ta negative anomalies, except tree samples (NE 11-13) which shows a $\mathrm{Pb}$ and $\mathrm{Sr}$ negative anomalies and $\mathrm{Ti}$ positive anomaly, and the minor group (NE $26,33,35)$ with a positive anomalies in Th and $\mathrm{La}$ and a negative anomalies in $\mathrm{Nb}-\mathrm{Ta}, \mathrm{Sr}$, except NE 26 which show a Sr positive anomaly (Figure 8(b)).

\section{Discussion}

The studied basic samples come from the Nemba Complex now considered synrift Neoproterozoic formation dated at $915 \pm 8 \mathrm{Ma}$ age SHRIMP gotten from meta-gabbro zircon, [5] and associated with volcanic (rhyolite) and volcano sedimentary rocks of Kakamoeka Subgroup whose equivalent called Mayumbian Group is dated in DRC between $920 \pm 8 \mathrm{Ma}$ and $917 \pm 14 \mathrm{Ma}$ [7].

\subsection{Petrogenesis}

\subsubsection{Magmatic Signature}

Major elements data (Table 1) shows a low potassic subalkaline affinity of the samples. [26] [29] diagrams (Figure 4) plots samples in their majority in tholeiite domain. These rocks are andesitic basalts and sub-alkaline basalts. [5] describes basalts of Nemba like sub-alkaline tholeiite.

\subsubsection{Crustal Contamination}

The LOI data of the geochemistry analysis are low to medium and shows that rocks are a weak weathering. Nevertheless the rocks contain a vein filled by quartz, feldspar and calcite which could characterize a hydrothermal weathering in relation to the deformation. This possibility is showing by the little migmatite zone seen on the outcrop (Figure 2(e)). Just has, geochemistry diagrams of studied meta-basalts displays a possible contamination of magmas by post-magmatic process. 


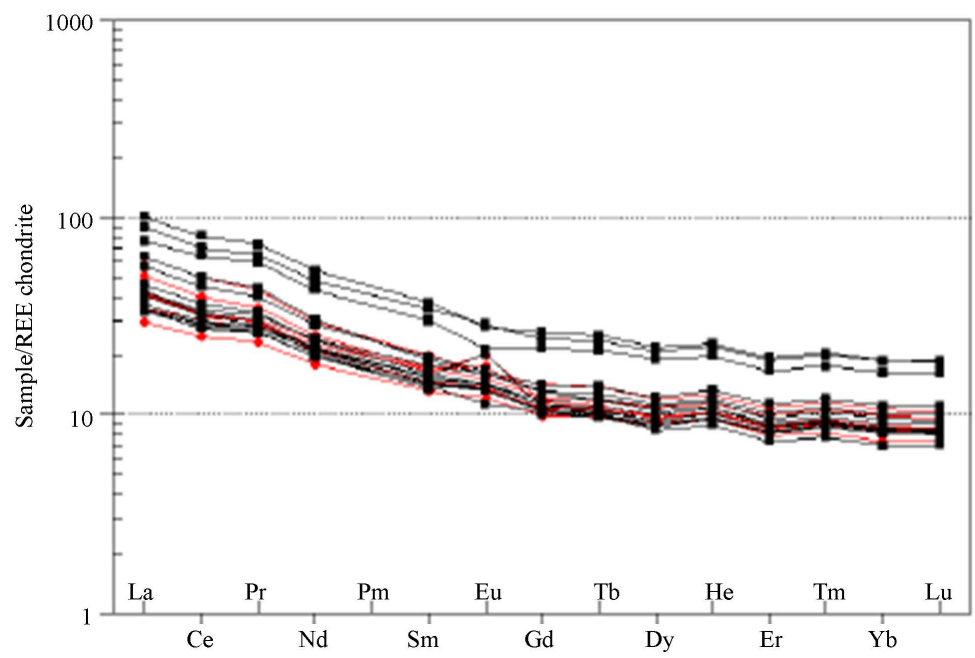

(a)

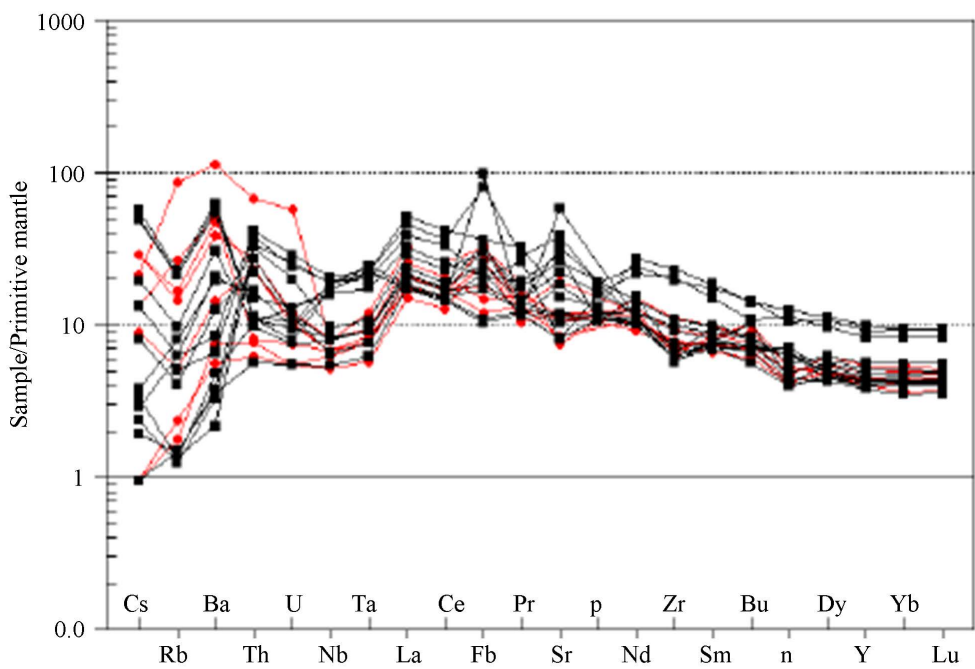

(b)

Figure 8. Spectrum of Rare Earth elements of Nemba Complex: a) normalized to chondrites of Nakamura (1974); b) normalized to the primitive mantle of McDonough and Sun (1995).

Precambrian meta-basalts are supposed to be products by magma from the enriched mantle, modified by the crustal contamination [34] [35] [36]. The basic rocks affected by the crustal contamination during subduction process may have negative anomalies in $\mathrm{Nb}-\mathrm{Ta}$ and a positive anomaly in $\mathrm{Sr}$ with a spectrum of enriched LREE [37] [38] [39] [40]. This is the case of Nemba meta-basalts which displays negatives anomalies in $\mathrm{Nb}-\mathrm{Ta}, \mathrm{Ti}$ and positives anomalies in $\mathrm{Sr}$ and $\mathrm{Ba}$ which point out the possibility of crustal contamination. This hypothesis is confirmed in $\mathrm{Nb} / \mathrm{Yb}$ vs $\mathrm{Th} / \mathrm{Yb}$ diagram of [31] (Figure 7(b)) which shows a weak dispersion of samples. Here samples are distributed between Arc volcanic and MORB domains and follow a tendency which indicate the crustal contamination (CA). This modification could be owned to the metasomatism of the mantle by 
process linked to subduction event. The contamination is also revealed by hydrothermal weathering characterized by the presence of fluids deposited quartz, feldspar and calcite in the vein crosscutting the rocks. Hydrothermal weathering is also described by [5] in the Nemba Complex. We can conclude that Nemba basalts are derived from primitive mantle (PM).

The spectrum of normalized multi-elements at primitive mantle of [33] displays (Figure 8(b)) a melting of enriched crustal component on showing negative anomalies in $\mathrm{Nb}-\mathrm{Ta}$ and $\mathrm{Ti}$ and positive anomalies in $\mathrm{Ba}$ and $\mathrm{Sr}$, typical of continental rocks. Impoverishment is high pronounced in the basic andesite. Tholeiite samples mark a difference with the calc-alkaline samples by the lower concentration in Th rendering the evolution from tholeiitic tendency to calc-alkaline tendency. The spectrum of multi-elements is characteristic of continental rocks with a lithospheric origin and is in agreement with a filiation of tholeiitic/calc-alkaline transition.

\subsection{Geodynamic context}

The $\mathrm{Zr}$ vs. Ti diagram of [30] displays that a majority of samples plots in the Arc Island lavas and MORB (Figure 7(a)). Tree samples are situated in the Within-Plate lavas. The meta-basalts with low-Ti are produced in intra-cratonic environment and the meta-basalts with high $\mathrm{Ti}$ are alimented by melting produced in the modified mantle in subduction across the oceanic crust [34]. [41] shows that active continental margin is characterized by basic rocks with high concentration in $\mathrm{K}, \mathrm{Sr}, \mathrm{Ba}$, HFSE. The samples of calc-alkaline group displays a high concentration in $\mathrm{K}, \mathrm{Sr}, \mathrm{Ba}$ and HFSE which suggest that they are take place in active continental margin. The geochemistry characteristic of Nemba meta-basalts point out that the region source has been modified by the implicate process in the lithospheric plate. This modification may be produced by the métasomatose of the mantle by the process linked at the subduction. In the active continental margins, the magma exchanges from the tholeiite to calc-alkaline and alkaline compositions in the fosse [41] [42] [43].

A similar picture is observed for the Nemba meta-basalts where the samples show tholeiite and calc-alkaline association. The Nemba meta-basalts could be supplied by a partial melting of sources enriched of Mantle with a crustal contamination of magma sources. Basalts and andesitic basalts of Nemba may be considered links to subduction type event with a contamination of the crust of Arc insular type. [5] suggests the assimilation of crustal component made of rocks associated with an insular arc build during eburnean event or anterior events. However, different works on the Nemba Complex and the magmatic associated rocks (alkali plutons and acid volcanic rocks) attribute there a rift context [5] [7] [11] [12] [44] although [10] have interpreted the basic rocks of Nemba like oceanic suture of the Mayombe belt. In Gabon, the equivalent of Nemba Complex is considered like MORB in mantle become poorer [45]. In DRC, basaltic sequence of Gangila of Zadinian Group dated between $930 \mathrm{Ma}$ 
and $920 \mathrm{Ma}$ is described like flood continental basalt put into place in rift context [6]. In Brazil, in Araçuài belt, the time between $920 \mathrm{Ma}$ and $900 \mathrm{Ma}$ will correspond to the development of continental rift in which were take place basalts of Macaùbas and Dom Silvério [46].

Data presented in this study shows that the majority of samples from Nemba situated between $\mathrm{Pk} 12$ and $\mathrm{Pk} 13$ have tholeiite signature and a few samples havecalc-alkaline signature but both have continental affinity. Isotopic data are necessary to complete chemical study in Nemba Complex to solve the geodynamic context of the west Congo fold belt.

\section{Conclusion}

The studied Nemba meta-basalts display a geochemistry composition in tholeiite majority. The evolution of the trace and rare earth elements shows a put into place in subduction context with a contamination by lithospheric crust of Insular Arc type. However, the association of the basic Nemba Complex with the plutonic rocks (Mfoubou and Mt Kanda granites) and effusive rocks (rhyolite and pyroclastic) of Kakamoeka Subgroup suggest the put into place in rift context. This assumption is in agreement with data gotten in the whole Araçuài-West Congo (A-WCO) belt.

\section{References}

[1] Heilbron, M. and Machado, N. (2003) Timing of Terrane Accretion in the Neoproterozoic-Eopaleozoic Ribeiraorogen (se Brazil). Precambrian Research, 125, 87-112. https://doi.org/10.1016/S0301-9268(03)00082-2

[2] Pedrosa-Soares, A.C. and Alkmin, F.F. (2011) How Many Rifting Events Preceded the Development of the Araçuai-West Congo Orogen? Geonomos, 19, 244-251.

[3] Pedrosa-Soares, A.C., Alkmin, F.F., Tack, L., Noce, C.M., Babinski, M., Silva, L.C. and Martins-Neto, M.A. (2008) Similarities and Differences between the Brazilian and African Counterparts of the Neoproterozoic Araçuai-West Congo Orogen. In: Pankhurst, R.J., Trouw, R.J., Brito Neves, B.B. and De Wit, M.J., Eds., West Gondwana: Pre-Cenozoic Correlations across the South Atlantic Region. Geological Society, London, 294, 153-172. https://doi.org/10.1144/SP294.9

[4] Affaton, P., Kalsbeek F., Boudzoumou, F., Trompette, R., Thrane, K. and Frei, R. (2015) The Pan-African West Congo Belt in the Republic of Congo (Congo Brazzaville): Stratigraphy of the Mayombe and West Congo Supergroups Studied by Detrital Zircon Geochronology. Precambrian Research, 272, 185-202. https://doi.org/10.1016/j.precamres.2015.10.020

[5] Fullgraf, T., Callec, Y., Thiéblemont, D., Gloaguen, E., Charles, N., Le Métour, J., Prian, J.-P., Boudzoumou, F., Delhaye-prat, V. Moreau, F., Kebi-Tsoumou, S. and Ndiele, B. (2015) Notice explicative de la carte géologique de la République du Congo à 1/200 000, Feuille Dolisie. Éditions BRGM, 331 p.

[6] Tack, L. (1975) Etude pétrochimique de la Formation des Roches vertes de Gangila (Bas-Zaïre). Annales Société Géologique Belgique, 98, 229-249.

[7] Tack, L., Wingate, M.T.D., Liégois, J.-P., Fernandez-Alonso, M. and Deblond, A. (2001) Early Neoproterozoic Magmatism (1000-910 Ma) of the Zadinian and Mayumbian Groups (Bas-Congo): Onset of Rodinia Rifting at the Western Edge of 
the Congo Craton. Precambrian Research, 110, 277-306.

https://doi.org/10.1016/S0301-9268(01)00192-9

[8] Boudzoumou, F. and Trompette, R. (1988) La Chaîne Ouest-Congolienne au Congo (Afrique Equatoriale) : un socle polycyclique charrié sur un domaine subautochtone formé par l'aulacogène du Mayombe et le bassin de l'Ouest-Congo. Bulletin Société Géologique de France, 8, 889-896. https://doi.org/10.2113/gssgfbull.IV.6.889

[9] Franssen, L. and André, L. (1988) The Zadinian Group (Late Proterozoic, Zaïre) and Its Bearing on the Origin of the West-Congo Orogenic Belt. Precambrian Research, 38, 215-234. https://doi.org/10.1016/0301-9268(88)90003-4

[10] Vellutini, P., Rocci, V., Vicat, J.P. and Gioan, P. (1982) Mise en évidence de complexes ophiolitiques dans la chaïne du Mayombe (Gabon-Angola) et nouvelle interpretation géotectonique. PrecambrianResearch, 22, 1-21.

https://doi.org/10.1016/0301-9268(83)90056-6

[11] Djama, L.M. (1988) Le massif granitique de Mfoubou et le socle métamorphique de Guéna (Chaîne du Mayombe-Congo). Pétrologie-Géochimie-Géochronologie. Thèse 3ème cycle, Université de Nancy I, 175 p.

[12] Djama, L.M., Leterrier, J. and Michard, A. (1992) Pb, Sr and Nd Isotope Study of the Basement of the Mayumbian Belt (Guena Gneisses and Mfoubou Granite, Congo): Implications for Crustal Evolution in Central Africa. Journal of African Earth Sciences, 14, 227-237. https://doi.org/10.1016/0899-5362(92)90100-Q

[13] Maurin, J.-C., Boudzoumou, F., Djama, L-M., Gioan, P., Michard, A., Mpemba-Boni, J., Peucat, J.-J., Pin, C. and Vicat, J.-P. (1991) La chaîne protérozoïque ouest-congolienne et son avant-pays au Congo: Nouvelles données géochronologiques et structurales, implications en Afrique centrale. Comptes Rendus de l'Académie des Sciences, 312, 1327-1334.

[14] Delhal, J. and Ledent, D. (1976) Age et évolution comparée des gneiss migmatitiques pré-zadiniens des régions de Boma et de Mpozo-Tombagadio (Bas-Zaïre). Annales de la Société géologique de Belgique, 99, 165-187.

[15] Delhal, J. and Ledent, D. (1978) Données géochronologiques dans la région de Matadi (Zaïre) relatives à la syénite de la Mpozo et aux métarhyolites. Rapport annuel, Musée Royal de l'Afrique Central, Département de Géologie et de Minéralogie, Tervuren, 99-110.

[16] Cahen, L., Delhal, D. and Ledent, D. (1978) Etudes géochronologiques dans la région de Boma (Zaïre): Le massif de Luki-Temvo, le granite de la Mao et le granite pegmatoïde de cul de Boma. Rapport annuel, Département Géologie Minière Musée Royal Afrique Central, Tervuren, 81-97.

[17] Cahen, L., Kröner, A. and Ledent, D. (1979) The Age of the Vista Alegre Pluton and Its Bearing on the Reinterpretation of the Precambrian Geology of Northern Angola. Annales de la Société géologique de Belgique, 102, 265-275.

[18] Mpemba-Boni, J. (1990) Contribution à l'étude du magmatisme ante-panafricain de la chaîne du Mayombe. L'exemple du massif de Les Saras (SW du Congo Afrique Centrale). Pétrologie structurale-Géochimie-Géochronologie. Thèse $3^{\text {ème }}$ cycle, Université de Nancy, France, 335 p.

[19] Dadet, P. (1969) Notice explicative de la carte géologique de la république du Congo-Brazzaville au 1/500 000. Mémoires du Bureau de Recherches Géologiques et Minières, Orléans, 70, $103 \mathrm{p}$.

[20] Hossie, G. (1980) Contribution à l'étude structurale de la chaîne Ouest-congolienne (Pan-Africaine) dans le Mayombe congolais. Thèse 3ème cycle. Montpellier, 124 p. 
[21] Boudzoumou, F. (1986) La chaîne panafricaine ouest-congolienne et son avant-pays au Congo (Afrique): Relations avec le Mayombe; Sédimentologie des séquences d'âge ProtérozoiqueSupéieur. Thèse $3^{\text {eme }}$ Cycle, Université d'Aix-Marseille III, 220 p.

[22] Cosson, J. (1955) Notice explicative sur les feuilles Pointe-Noire et Brazzaville. Carte géologique de reconnaissance au 1/500 000. Bulletin Direction des Mines et de la Géologie, Afrique Equatoriale Française, 56 p.

[23] Nicolini, P. (1959) Le Synclinal de la Nyanga (zone de la boucle du Niari). Contribution à l'étude de la minéralisation stratiforme du Moyen Congo. Bulletin Direction des Mines et de la Géologie, Afrique Equatoriale Française, 10, 178 p.

[24] Hossié, G. and Caby, R. (1979) Unicité des déformations et du métamorphisme d'agepan-africain dans la chaîne ouest-congolienne. Résumés, $10^{\circ}$ Colloque de Géologie Africaine, Montpellier, 25-27 avril 1979, 50-51.

[25] Cosma, L., Lapierre, H., Jaillard, E., Laubacher, G., Bosch, D., Desmet, A., Mamberti, M. and Gabriele, P. (1998) Pétrolographie et géochimie des unités magmatiques de la cordilliere occidentale d'Equateur ( $\left.0^{\circ} 30^{\prime} \mathrm{S}\right)$ : Implications tectoniques. Bulletin Société Géologique de France, 169, 739-751.

[26] Jensen, L.S. (1976) A New Cation Plot for Classifying Subalkalic Volcanic Rocks. Ontario Division Mines, Miscellaneous Paper, 66, 22 p.

[27] Peccerillo, A. and Taylor, S.R. (1976) Geochemistry of Eocene Calc-Alkaline Volcanic Rocks from the Kastamonu Area, Northern Turkey. Contribution to Mineralogy and Petrology, 58, 63-81. https://doi.org/10.1007/BF00384745

[28] Cox, K.G., Bell, J.D. and Pankhurst, R.J. (1979) The Interpretation of Igneous Rocks. Allen and Unwine, London, $450 \mathrm{p}$.

https://doi.org/10.1007/978-94-017-3373-1

[29] Winchester, J.A. and Floyd, P.A. (1977) Geochemical Discrimination of Different Magma Series and Their Differentiation Products using Immobile Elements. Chemical Geology, 20, 325-343. https://doi.org/10.1016/0009-2541(77)90057-2

[30] Pearce, J.A. (1982) Trace Element Characteristics of Lavas from Destructive Plate Boundaries. In: Andesites, Ed., Thorpe R S Chichester, Wiley, Hoboken, 525-548.

[31] Pearce, J.A. (2008) Geochemical Fingerprinting of Oceanic Basalts with Applications to Ophiolite Classification and the Search for Archean Oceanic Crust. Lithos, $100,14-48$.

[32] Nakamura, N. (1974) Determination of REE, Ba, Fe, Na, and K in Carbonaceous and Ordinary Chondrites. Geochimica and Cosmochimica Aeta, 38, 757-775. https://doi.org/10.1016/0016-7037(74)90149-5

[33] McDonough, W.F. and Sun, S.S. (1995) The Composition of the Earth. Chemical Geology, 120, 223-253. https://doi.org/10.1016/0009-2541(94)00140-4

[34] Ghose, N.C., Mukherjee, D. and Chatterjee, N. (2005) Plume Generated Mesoproterozoic Mafic-Ultramafic Magmatism in the Chotanagpur Mobile Belt of Eastern Indian Shield Margin. Journal Geology Society India, 66, 725-740.

[35] Ghose, N.C. and Chatterjee, N. (2008) Petrology, Tectonic Setting and Source of Dykes and Related Magmatic Bodies in Chotanagpur Gneissic Complex, Eastern India. In: Srivastava, R.K., Sivaji, C. and Chalapathi Rao, N.V., Eds., Indian Dyke: Geochemistry, Geophysics and Geochronology, Narosa Publishing House Pvt Ltd., New Delhi, 471-493.

[36] Kumar, A. and Ahmad, T. (2007) Geochemistry of the Mafic Dykes in Parts of Chotanagpur Gneissic Complex: Petrogenetic and Tectonic Implications. Geochemical Journal, 41, 173-186. https://doi.org/10.2343/geochemj.41.173 
[37] Crawford, A.J. (1989) Boninites and Related Rocks. Unwin Hyman, London, 465 p.

[38] Tatsumi, Y. and Eggins, S.M. (1995) Subduction Zone Magmatism. Blackwell, Oxford, $211 \mathrm{p}$.

[39] Zhao, J.H. and Zhou, M.F. (2007) Geochemistry of Neoproterozoic Mafic Intrusions in the Panzhihua District (Sichuan Province, SW China): Implications for Subduction-Related Metasomatism in the Upper Mantle. Precambrian Research, 152, 27-47. https://doi.org/10.1016/j.precamres.2006.09.002

[40] Ernst, R.E., Srivastava, R., Bleeker, W. and Hamilton, M. (2010) Precambrian Large Igneous Provinces (LIPs) and Their Dyke Swarms: New Insights from High-Precision Geochronology Integrated with Paleomagnetism and Geochemistry. Precambrian Research, 183, 379-668. https://doi.org/10.1016/j.precamres.2010.09.001

[41] Wilson, M. (1989) Igneous Petrogenesis. Unwin Hyman, London, 466 p. https://doi.org/10.1007/978-1-4020-6788-4

[42] Rickwood, P.C. (1989) Boundary Lines within Petrologic Diagrams Which Use Oxides of Major and Minor Elements. The Lithos, 22, 247-263.

[43] Hooper, P.R. (1994) Sources of Continental Flood Basalts: The Lithospheric Component. In: Subbarao, K.V., Ed., Volcanism, Wiley Eastern Limited, New Delhi, 29-53.

[44] Vicat, J.P. and Pouclet, A. (2000) Palaeo- and Neoproterozoic Granitoids and Rhyolites from the West Congolian Belt (Gabon, Congo, Cabinda, North Angola): Chemical Composition and Geotectonic Implications. Journal of African Earth Sciences, 31, 597-617. https://doi.org/10.1016/S0899-5362(00)80009-3

[45] Thiéblemont, D., Castaing, C., Billa, M., Bouton, A. and Préat, A. (2009) Notice Explicative de la carte géologique et des ressources minérales de laRépublique Gabonaise à 1/1000000. Programme Sysmin 8 ACP GA 017, Ministère des Mines, du Pétrole, des Hydrocarbures. Direction Générale des Mines et de la Géologie, 384 p.

[46] Alkmim, F.F., Marshak, S., Pedrosa-Soares, A.C., Peres, G.G., Cruz, S.C.P. and Whittington, A. (2006) Kinematic Evolution of the Araçuai-West Congo Orogen in Brazil and Africa: Nutcracker Tectonics during the Neoproterozoic Assemby of Gondwana. Precambrian Research, 149, 43-64.

https://doi.org/10.1016/j.precamres.2006.06.007 